\title{
Parton content of the real photon: astrophysical implications
}

\section{Alikhanov ${ }^{\text {a }}$}

Institute for Nuclear Research of the Russian Academy of Sciences, 60th October Anniversary pr. 7a, Moscow 117312, Russia

Received: 26 January 2009 / Published online: 5 March 2009

(C) Springer-Verlag / Società Italiana di Fisica 2009

\section{Erratum to: Eur. Phys. J. C (2008) 56: 479-482 DOI 10.1140/epjc/s10052-008-0684-5}

We have recently calculated the inclusive cross section of the reaction $v \gamma \rightarrow W^{+} X$ at the resonance region within the framework of the parton model [1]. We have considered a subprocess of annihilation of the neutrino with a positron coming from the photon into the $W^{+}$boson.

The cross section of this subprocess near the resonance can be parameterized by the Breit-Wigner formula:

$\hat{\sigma}(x s)=24 \pi \frac{\Gamma_{\mathrm{i}} \Gamma}{\left(x s-m_{W}^{2}\right)^{2}+m_{W}^{2} \Gamma^{2}}$,

where $x$ is the fraction of the photon's momentum carried by the positron, $s$ is the total center-of-mass energy squared of the $v \gamma$ reaction, $m_{W}$ is the mass of the $W^{+}$boson, $\Gamma_{\mathrm{i}}$ is the partial width of the initial channel (the partial width for the decay $W^{+} \rightarrow v_{e} e^{+}$), and $\Gamma$ is the total decay width of $W^{+}$.

This cross section is two times greater than that given in [1]. This is because in (1) we have correctly treated the possible spin states of the initial particles.

In averaging the absolute square of the corresponding amplitude over the initial spin states, one has to pay attention to the fact that positrons may appear in two spin states, while the neutrino has only one state of negative helicity.

Thus, we write the corrected cross section of the reaction $v \gamma \rightarrow W^{+} X$ :

$$
\begin{aligned}
\sigma(s)= & \frac{8}{3} \frac{\alpha \Gamma^{2}}{s^{3}}\left[2 s+\frac{s^{2}-2 m_{W}^{2}\left(s+\Gamma^{2}-m_{W}^{2}\right)}{\Gamma m_{W}}\right. \\
& \times\left(\arctan \frac{s-m_{W}^{2}}{\Gamma m_{W}}+\arctan \frac{m_{W}}{\Gamma}\right) \\
& \left.+\left(s-2 m_{W}^{2}\right) \ln \frac{\Gamma^{2} m_{W}^{2}+m_{W}^{4}}{\Gamma^{2} m_{W}^{2}+\left(s-m_{W}^{2}\right)^{2}}\right] \ln \frac{s}{m_{e}^{2}},
\end{aligned}
$$

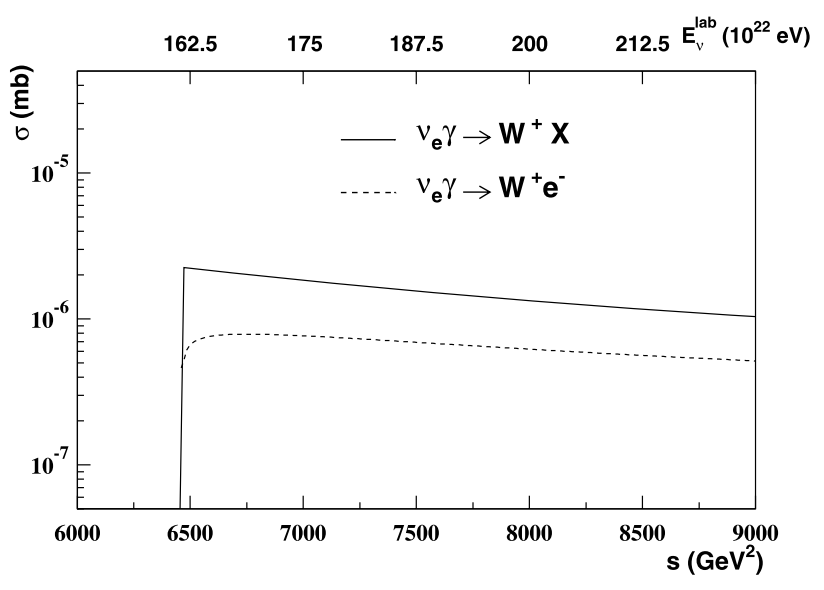

Fig. 1 Dependence of the cross section of the inclusive reaction $v_{e} \gamma \rightarrow W^{+} X$ on $s$ in the resonance region (solid curve). The same, calculated for the closely related reaction $v_{e} \gamma \rightarrow W^{+} e^{-}$[2], is shown by the dashed curve. The laboratory energy of the neutrino $E_{v}^{\text {lab }} \simeq s / 4 \varepsilon$ is calculated at $\varepsilon=10^{-3} \mathrm{eV}$

where $\alpha$ is the fine-structure constant. One can obtain this result by multiplying equation (6) from [1] by a factor 2 .

The dependence of this cross section on $s$ is shown in Fig. 1 in comparison with calculations of the closely related process $v \gamma \rightarrow W^{+} e^{-}$carried out in [2]. All the fixed parameters are the same as in [1].

One can see that in the considered range of variation of $s$ the corrected cross section is about two times higher than the one from [2].

\section{References}

1. I. Alikhanov, Eur. Phys. J. C 56, 479 (2008)

2. D. Seckel, Phys. Rev. Lett. 80, 900 (1998)

a e-mail: ialspbu@gmail.com

The online version of the original article can be found under doi:10.1140/epjc/s10052-008-0684-5. 